\title{
Estimation of Power Losses on Radial Feeder Using Minimum Electrical Measurements and Differential Evolution Method
}

\author{
${ }^{1}$ Marinko Barukcic, ${ }^{2 *}$ Srete Nikolovski, ${ }^{3}$ Zeljko Hederic \\ Faculty of Electrical Engineering, J.J. Strossmayer University of Osijek, Croatia \\ ${ }^{1}$ marinko.barukcic@etfos.hr, ${ }^{2}$ srete.nikolovski@etfos.hr. ${ }^{3}$ zeljko.hederic@etfos.hr
}

\begin{abstract}
The topic of research in this paper is the determination of losses on a radial distribution feeder at a certain moment based on measurements of input complex power in the feeder and voltages in the farthest network node and power flow calculation. The presented method of DE used to find a virtual load of each node is based on the defined objective function. The value of the objective function using DE algorithm is obtained by calculation of power flow. The virtual load thus obtained is then used in the calculation of power flows on the basis of which total losses in a network are determined. The proposed method attempts to overcome the problems arising from the uncertainty of node loads in the network at some point in the evaluation of network losses. Evaluation of the proposed method was carried out by performing tests on the IEEE 123 node test feeder.
\end{abstract}

Keywords: Apparent power, Differential Evolution, Power Losses Estimation, Radial Feeder, Voltage.

\section{Introduction}

It was important for the distributors to know the total losses in the distribution networks. The issue of loss estimation has long been treated in the literature and there are many papers on this subject. The literature shows different methods of losses estimation in the distribution network. In [1] a method for losses estimation on the basis of knowing load of certain network components is shown. It is necessary to know peak and real power that goes through the component in this method. Often used are methods where losses are estimated with loss factor which depends on load factor. Examples of these methods can be found in [2] and [3]. Methods where explicit expressions are used for losses estimation are shown in [4], [5] and [6]. In these methods the entire calculation of power flow is not performed. The time of calculation is considerably diminished. In [7] a method based on power measurement injected in the network is shown, voltage on HV and MV sides of HV/MV transformers and current in main feeder. Models based on theory of fuzzy sets have been developed lately. In these methods losses are estimated on the basis of network total load by applying fuzzy regression. Analytic expression determining, with fuzzy numbers, dependence of loss on load is based on measured data for total load and total losses. These methods are shown in [8], [9] and [10]. Top-Down/Bottom-Up method for losses estimation is shown in [11]. Here first the larger group of distribution feeders is grouped (clustered) into smaller groups with similar feeders. According to method [12] the network is firstly separated into groups with similar feeders and in each group a representative feeder is determined. After a certain time all loads are measured on these feeders. Then on the basis of measured data the losses are calculated. The calculated losses are compared with the losses estimated with the existing methods. Losses on other feeders in the 
International Journal of

Soft Computing And Software Engineering (JSCSE)

e-ISSN: 2251-7545

Vol.2,No.4, 2012

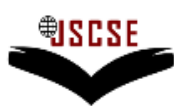

DOI: 10.7321/jscse.v2.n4.1

group are estimated with the method that gives the best results comparing to losses obtained by measuring. Loss calculation in the network is performed by calculation of power flows. To do that it is necessary to know the network data: nominal voltage, lines data, network configuration and node loads. Regarding load, usually it is the easiest way to get the data on installed (nominal) load power.

Exact loads in nodes at some point can exclusively be obtained by measuring. A larger number of consumers is usual in distribution networks. Because of that the measurement of all loads causes great financial and technical effort. Commonly the loads in distribution networks are estimated for the needs of different calculations. The simplest way is to multiply nominal loads of each node with ratio of total current and nominal power of the entire network. However, the loads of all nodes are not changing in the same ratio simultaneously. Because of that this way of load estimation often does not correspond to real network state. Other way is to estimate current node load with load curves. Load curves are attained from measured history for different consumer types. These curves can be measured on yearly (load per months), monthly (load per days) and daily (load per hours) basis. However, in this case it is not entirely safe that the observed load changes in time completely the same like the previously measured for some consumer type. This is clearly expressed in the consumers of household category. Also, these curves are mostly obtained by measuring the total power of the entire network, not each consumer separately. Due to this unreliability when determining node loads even the calculated losses can differ from the real ones.

In this paper the intention was to overcome these problems by defining certain combinations of current loads in network nodes. This way the load estimation is performed with minimal number of measured data. An objective function which contains differences between measured and calculated data is defined, and the observed problem is solved with evolutionary algorithm. By solving the optimization problem a certain combinations of network loads are obtained. We called the node loads which are obtained in such a way virtual loads because they do not necessarily correspond to real node loads. We tried to prove that these virtual loads give approximately the same losses to the ones calculated for real loads by empirical way and simulation performances.

\section{Defining of the optimization problem}

The idea for the problem solution is based on the analysis of complex power balance and influence of node loads on losses and voltage in the network. Network node load can vary during the times from zero value to its nominal or peak value. The total network load is equal to the sum of all node loads in certain moment. For a certain total network load that is smaller than the peak load, a certain number of different node load combinations can exist. It can be concluded that different combinations of node power, which give approximately the same total power, in general case give different losses. The question emerges here: do two (or more) combinations which give approximately the same total power and total losses in the network exist (Figure 1)? If they exist then for each real loads combination can be written:

$S_{\text {in }}=S_{l}+\sum_{i=1}^{n} S n_{i} \approx S_{l}^{j}+\sum_{i=1}^{n} S n_{i}^{j}=S_{i n}^{j}$

Where: $S_{i n}$ - complex power injected into the radial feeder, $S_{l}$ - total complex losses of feeder, $S n_{i}-$ complex power of load in the $i^{\text {th }}$ node, $S_{i n}^{j}-$ complex power injected into the radial feeder for the $j^{\text {th }}$ combination of loads, $S_{l}^{j}-$ total complex losses of feeder for the $j^{\text {th }}$ combination of virtual loads, $S n_{i}^{j}-$ complex power of load in the $i$-th node for the $j^{\text {th }}$ combination of loads (can be different from corresponding real load) and $n$ - number of nodes with load. Index $j$ in (1) marks some combination of virtual loads if there is more combinations of virtual loads for same injected power.

Part in $(1)$ on the left side of the sign $\approx$ represents the real total power, losses and load in the network. Part in $(1)$ on the left side of the sign $\approx$ represents the sign balance for certain node loads combination which do not have to be the same as the real one. Sum elements in (1) are complex loads of nodes in the network. The assumption is that the loads are in the same nodes. It is necessary to point out that the sums on the right and left side of sign $\approx$ in $(1)$ are approximately the same, but the corresponding elements of 
every sum do not have to be equal. Different combinations of network node loads also give different voltage in network nodes. The idea is to set an optimization problem which contains known (measured) total network power and known (measured) voltage in the last network node.

\subsection{Objective function}

Objective function of optimization problem in the general shape is set as:

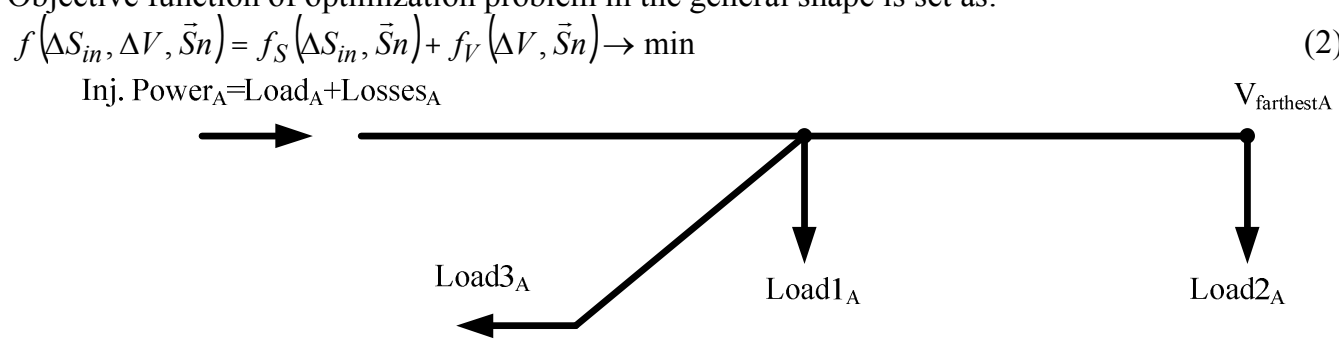

Case A: real state of the network

Are there such: $\operatorname{Load} 1_{\mathrm{B}} \neq \operatorname{Load} 1_{\mathrm{A}}, \operatorname{Load} 2_{\mathrm{B}} \neq \operatorname{Load} 2_{\mathrm{A}}, \operatorname{Load} 3_{\mathrm{B}} \neq \operatorname{Load} 3_{\mathrm{A}}$, so that: Inj. Power $\mathrm{B} \approx$ Inj. Power $_{\mathrm{A}}, \operatorname{Load}_{\mathrm{B}} \approx \operatorname{Load}_{\mathrm{A}}, \operatorname{Losse}_{\mathrm{B}} \approx \operatorname{Losses}_{\mathrm{A}}$ and $\mathrm{V}_{\text {farthest }} \approx \mathrm{V}_{\text {farthestA }}$ ?

Inj. Power $_{B}=\operatorname{Load}_{B}+\operatorname{Losses}_{B}$

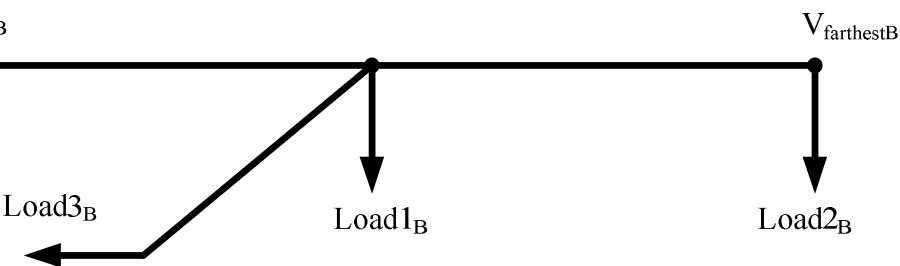

Case B: virtual state of the network

Figure 1. Preview of the idea

Where $\vec{S} n=\left[S n a_{1}, S n b_{1}, S n c_{1}, \ldots S n a_{i}, S n b_{i}, S n c_{i} \ldots S n a_{n}, S n b_{n}, S n c_{n}\right]^{\mathrm{T}}$ is vector of loads, combination of all complex phase loads in vector form, $\Delta S_{\text {in }}$ is a difference between measured and calculated injected complex powers and $\Delta V$ is a difference between measured and calculated voltages of the last network node. Part $f_{S}$ in (2) refers to the part of objective function which contains the difference of total powers in itself, and $f_{V}$ on part that contains difference of voltages in the last network node. Below are details of certain parts of objective function (2). Absolute value of differences between the measured and calculated (for virtual loads) injected active power is:

$$
\Delta P_{k}(\vec{S} n)=\left|\operatorname{Re}\left\{S m_{k}-S c_{k}(\vec{S} n)\right\}\right|
$$

Where, $S m_{k}$ and $S c_{k}$ are total measured complex power injected into the $k^{\text {th }}$ phase of the feeder and total calculated complex power injected into the $k^{\text {th }}$ phase of the feeder for some combinations of virtual loads.

Furthermore, for the absolute values of the difference between imaginary parts of the measured and calculated complex power of the $k$ - th phase, the expression is: 


$$
\Delta Q_{k}(\vec{S} n)=\left|\operatorname{Im}\left\{S m_{k}-S c_{k}(\vec{S} n)\right\}\right|
$$

$\mathrm{Re}$ and Im in (3) and (4) mark real i.e. imaginary part of complex powers.

In (3) and (4), the calculated complex power $(S c)$ is obtained for virtual node loads which are formed during the performance of DE. The measured power in (3) and (4) is carried out for unknown real node loads.

In order to determine the proper impact of the absolute difference between active and reactive injected power on the value of the objective function, it has been defined as:

$$
f_{S}\left(\Delta S_{i n}, \vec{S} n\right)=\sum_{k=a, b, c}\left\{\exp \left(\Delta P_{k}(\vec{S} n) / D_{P}\right)+\exp \left(\Delta Q_{k}(\vec{S} n) / D_{Q}\right)\right\}
$$

Calculation of the difference of injected active and reactive power according to (3) and (4) gives results which, however, do not offer solutions of the same quality. According to (5), the scaling coefficients $D_{P}$ and $D_{Q}$ are introduced on the basis of which the behavior of the objective function adjusts to the values measured in the injected power. $D_{P}$ and $D_{Q}$ coefficients equalize the value of members in (5) which relate to active and reactive power. The following expressions are used to determine the coefficients $D_{P}$ and $D_{Q}$ :

$$
D_{P}=0.01 \cdot P_{N n} ; \quad D_{Q}=0.01 \cdot Q_{N n}
$$

Where $P_{N n}$ and $Q_{N n}$ are injected nominal real and reactive power. Coefficient 0.01 which multiplies the nominal active and reactive power (6) is introduced due to equalizing of numerical values of objective function values $f_{V}$ and $f_{S}$. Also this coefficient enables that the changes (lowering or increasing) of value $f_{V}$ and $f_{S}$ caused by changing objective function variables are equalized. The value of this coefficient is changed by that and the reached conclusion is that for the stated function forms $f_{S}(5)$ and $f_{V}$ (7) in general the 0.01 value fits the best. In (5)-(8) active and reactive power are expressed in $\mathrm{kW}$ and kvar.

For $f_{V}$ of objective function the following expression is used:

$$
f_{V}(\Delta V, \vec{S} n)=\sum_{k}\left\{\exp \left(V_{n} \cdot\left|V_{c k}(\vec{S} n)-V_{m k}\right|\right)\right\} \rightarrow \min
$$
[p.u.].

In (7) the nominal phase voltage $V_{n}$ is in [V], and calculated and measured voltages $V_{c k}$ and $V_{m k}$ are in

Function forms $f_{S}(5)$ and $f_{V}(7)$ are determined experimentally by performing simulation for more different forms. The amount of node load in the network can vary from a minimum value (may be 0 ) to the peak or installed nominal load values. By taking this constraint into account, the optimization problem is solved as:

$$
\begin{aligned}
& f\left(\Delta S_{i n}, \Delta V, \vec{S} n\right)=f_{S}\left(\Delta S_{i n}, \vec{S} n\right)+f_{V}(\Delta V, \vec{S} n)= \\
& \sum_{k}\left\{\exp \left(\Delta P_{k}(\vec{S} n) / D_{P}\right)+\exp \left(\Delta Q_{k}(\vec{S} n) / D_{Q}\right)\right\}+\sum_{k}\left\{\exp \left(V_{n} \cdot\left|V_{c k}(\vec{S} n)-V_{m k}\right|\right)\right\} \rightarrow \min \\
& \text { subject to }: 0 \leq S n_{i} \leq S n_{i, \max }
\end{aligned}
$$

As seen from (3), (4), (5), (7) and (8), the objective function variables are complex power of phase node loads in all network nodes. Value of global objective function minimum is 0 . This value corresponds to real node loads. For objective function (8) with evolutionary algorithm a values of node loads (objective function variables) which give the smallest value of objective function are searched. In the end by calculation of power flows the losses in network are defined from node loads which are obtained in such a way.

\section{Finding node loads by differential evolution}

Differential Evolution (DE) belongs to a group of evolutionary algorithms and is often used to solve 
optimization problems. The basic algorithm of Differential Evolution is simple and consists of the following steps [13]:

Step 1 Create a population of $N i$ vectors in the starting generation.

Step 2 For each vector in the population repeat steps 3 to 6 .

Step 3 Create a mutant vector.

Step 4 Create a trial vector by combining the mutant vectors and target vector.

Step 5 Check whether the value of the objective function for the trial vector is less than or equal to the value of the objective function of a vector in the current population.

Step 6 Replace the existing vector with trial vector if the condition in step 5 is fulfilled.

Step 7 Replace previous population of vectors with a new population obtained by performing steps 3 to 6 .

Step 8 Repeat steps 2 to 7 until the criteria for stopping the algorithm is not fulfilled.

Step 9 As a solution of the problem, print the vector with the smallest objective function value in the last generation.

The following describes specific actions required for the application of Differential Evolution for the considered problem.

\subsection{Initial population}

Population in the evolutionary algorithms is a group of individuals from the problem solution space. The individual represents a group of values of objective function variables. The individual is usually mathematically shown in the form of vector or data series. The elements of individual vector are variables of objective function. For set optimization problem (8) the individual can be shown with the vector whose elements are complex phase load powers in nodes:

$$
\vec{S} n=\left[S n a_{1}, S n b_{1}, S n c_{1} \cdots, S n a_{i}, S n b_{i}, S n c_{i} \cdots, S n a_{m}, S n b_{m}, S n c_{m}\right]^{T}
$$

Where $S n_{i}$ is a complex load in $i^{\text {th }}$ node for $a, b$ and $c$ phases and $m$ the number of nodes with the loads. Individual population can be mathematically shown in matrix shape as:

$$
P O P=\left[\begin{array}{cccccc}
S n a_{1}^{1} & S n a_{1}^{2} & \cdots & S n a_{1}^{j} & \cdots & S n a_{1}^{p} \\
S n b_{1}^{1} & S n b_{1}^{2} & \cdots & S n a_{1}^{j} & \cdots & S n a_{1}^{p} \\
\vdots & \vdots & \cdots & \vdots & \cdots & \vdots \\
S n a_{i}^{1} & S n a_{i}^{2} & \cdots & S n a_{i}^{j} & \cdots & S n a_{i}^{p} \\
\vdots & \vdots & \cdots & \vdots & \cdots & \vdots \\
S n c_{m}^{1} & S n c_{m}^{2} & \cdots & S n c_{m}^{j} & \cdots & S n c_{m}^{p}
\end{array}\right]=\left[\begin{array}{llllll}
\overrightarrow{S n^{1}} & \overrightarrow{S n^{2}} & \cdots & \overrightarrow{S n^{j}} & \cdots & S^{p} \\
& & & & &
\end{array}\right]
$$

Where $p$ is a number of individuals in population i.e. population size.

If the influence of variable value on objective function is not known, usually the initial population is created by random selection of individuals from the solution space. In some cases, areas in solution space in which there could be a greater possibility of problem solution existence are known. In those cases the creation of initial population is performed by selection of individuals from those interesting areas. The losses in the distribution networks amount couple of percentages of the total network power. So the network load makes the majority of injected power in the network. On these grounds it can be concluded that in the observed optimization problem the interesting areas in solution space are those where the sum of all loads is close to the measured injected power. Because of that the individuals of initial population is created here in a way that the sum of all individual elements is equal to the power injected in the network. Due to diversity of genetic material in the population, the unit element values are selected randomly. It should be taken into account that the loads can move in limits from 0 to the nominal value. In the general case of nominal value of certain loads are not equal and can differ significantly. For the creation need of initial population individuals, with the condition that the sum of individual elements is 
equal to the injected power, the following procedure is used here:

Step 1: The nodes with loads are set in the series with random order selection.

Step 2: The values of coefficient $K$ ranging to $R$ to 1 are set with step $\Delta K . R$ is the ratio of injected network power and nominal network load. The step $\Delta K$ is calculated as:

$$
\Delta K=(1-R) /\left(N_{K}-1\right)
$$

Where $N_{K}$ is the number of coefficient value $K$. The number of coefficient value $K$ can be selected at will. The values of coefficient $K$ are calculated as:

$$
K_{i}=1-n \cdot \Delta K \quad \text { for } n=0,1,2,3, \cdots, N_{K}-1
$$

Step 3: The first load in sequence from Step 1 is set on nominal value multiplied with coefficient $K$.

Step 4: For other loads in sequence Step 3 is repeated until the sum of all previously determined loads is less then injected power.

Step 5: If the sum of all previously determined loads is higher then injected power, then the last determined load is equal to difference between injected power and sum of all previously determined loads.

Step 6: Steps 3-5 are repeated for all values of coefficient $K$ set in step 2.

By making initial population with this procedure a sufficient genetic diversity in the initial population is ensured. Also, with this the search of solution space in future generations DE is focused on interesting areas. If power injected into network is greater than nominal load of network this procedure will not be performed. The network is close to its nominal state, in this case. The number of individuals created with the described procedure is equal to the number of coefficient value $K\left(N_{K}\right.$ in Step 2). By implementing the described procedure $N$ times a size of the population is obtained which is equal to product $N$ and $N_{K}$. In Table 1 an example of described procedure implementation with numerical values for network example with 4 loads and $N_{K}=5$ is shown.

\begin{tabular}{|c|c|c|c|c|c|c|c|}
\hline \multirow{2}{*}{ Nominal node loads } & $L 1_{n}$ & $L 2_{n}$ & $L 3_{n}$ & $L 4_{n}$ & & & $\sum L$ \\
\hline & 50 & 30 & 70 & 100 & & & 250 \\
\hline Random loads order & \multicolumn{7}{|c|}{$L 2, L 3, L 1, L 4$} \\
\hline Injected network power $P_{\text {in }}$ & \multicolumn{7}{|c|}{100} \\
\hline Ratio $R=\operatorname{Pin} / \sum L$ & \multicolumn{7}{|c|}{0.4} \\
\hline Number of coefficients $K, N_{K}$ & \multicolumn{7}{|c|}{5} \\
\hline Step of $K, \Delta K=(1-R) /\left(N_{K^{-}}-1\right)$ & \multicolumn{7}{|c|}{0.15} \\
\hline \multirow{7}{*}{$\begin{array}{l}\text { Generating of initial } \\
\text { population }\end{array}$} & \multicolumn{5}{|c|}{ Load combinations } & \multirow{2}{*}{$\sum L$} & \multirow{2}{*}{$\begin{array}{l}\text { Initial population with five } \\
\text { individuals }\left[\begin{array}{llll}L 1 & L 2 & L 3 & L\end{array}\right]^{\mathrm{T}}\end{array}$} \\
\hline & values & $L 2$ & $L 3$ & $L 1$ & $L 4$ & & \\
\hline & 0.4 & 12 & 28 & 20 & 40 & 100 & {$\left[\begin{array}{lllll}20 & 12 & 28 & 40\end{array}\right]^{\mathrm{T}}$} \\
\hline & \begin{tabular}{|l|}
0.55 \\
\end{tabular} & 16.5 & 38.5 & 27.5 & 17.5 & 100 & $\left.\begin{array}{lllll}27.5 & 16.5 & 38.5 & 17.5\end{array}\right]^{\mathrm{T}}$ \\
\hline & 0.7 & 21 & 49 & 30 & 0 & 100 & {$\left[\begin{array}{lllll}30 & 2 & 1 & 49 & 0\end{array}\right]^{\mathrm{T}}$} \\
\hline & \begin{tabular}{|l|}
0.85 \\
\end{tabular} & 25.5 & 59.5 & 15 & 0 & 100 & {$\left[\begin{array}{lllll}15 & 25.5 & 59.9 & 0\end{array}\right]^{\mathrm{T}}$} \\
\hline & 1 & 30 & 70 & 0 & 0 & 100 & {$\left[\begin{array}{lllll}0 & 30 & 70 & 0\end{array}\right]^{\mathrm{T}}$} \\
\hline
\end{tabular}

Table 1. An example of initial population generating

\subsection{Mutant and trial vectors}

The mutant vector is made by combination of individuals (vectors) from the current population. There are several ways in which the mutant vectors are generated in DE. In the paper, the following expression for the mutant vector has been used [13]: 


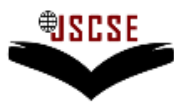

DOI: $10.7321 / j \operatorname{sese.v2.n4.1~}$

$$
\begin{aligned}
& \vec{S}_{g}^{j}=\vec{S}_{g}^{r 1}+F \cdot\left(\vec{S}_{g}^{r 2}-\vec{S}_{g}^{r 3}\right)+F \cdot\left(\vec{S}_{g}^{r 4}-\vec{S}_{g}^{r 5}\right) \\
& r l \neq r 2 \neq r 3 \neq r 4 \neq r 5
\end{aligned}
$$

The $S_{g}{ }^{r 1}$ is the base vector and $S_{g}{ }^{r 2}, S_{g}{ }^{r 3}, S_{g}{ }^{r 4}$ and $S_{g}{ }^{r 5}$ are difference vectors. The base vector can be chosen in different ways. For example, it is the current best population vector or linear combination of various vectors or a randomly chosen population vector. The base vector is a randomly chosen population vector, in the presented methode. Difference vectors are randomly selected once per base vector. The $F$ is DE parameter, called mutation factor, which controls the amplification of the difference between individuals and it is usually taken from the range $[0.1,1][13]$. The number of mutant vectors is equal to the number of individuals in the population, i.e. equal to the population size.

Elements of the trial vectors in DE are defined as [13]:

$$
S t_{g, i}^{j}= \begin{cases}S m_{g, i}^{j} & \text { if } \quad\left(\text { rand }_{i}[0,1) \leq C r\right) \\ S_{g, i}^{j} & \text { otherwise }\end{cases}
$$

Where $S t_{g, i}$ is the $i^{\text {th }}$ element of the $j^{\text {th }}$ trial vector for the current population. The $S_{g}^{j}$ is the $j^{\text {th }}$ individual (usually caled the target vector) from the current population and $S_{g, i}^{j}$ is the $i^{\text {th }}$ element of the $S_{g}^{j}$. The $\mathrm{Cr}$ is DE parameter, called crossover rate in the range [0,1] [13]. The random number rand $_{i}$ is evaluated for each element of the mutant vector. The trial vector is made for each mutant vector and population individual. According (14), the trial vector element is equal to the corresponding mutant vector elemen if $\mathrm{rand}_{i} \leq \mathrm{Cr}$. Otherwise it is equal to the corresponding element of the current population vector. The mutation and trial vectors performed mutation and recombination of individual elements (genes). For each trial vector, the objective function value is calculated. For mutant vectors, objective function values are not calculated.

\subsection{Selection and creation of the population in the next generation}

The new population in DE is created by comparing objective function values of the trial vector and corresponding current population individual (target vector). Selection in the Differential Evolution is very simple and is done according to the following criterion [13]:

$$
\vec{S}_{g+1}^{j}= \begin{cases}\vec{S}_{g}^{j} & \text { if }\left(f\left(\vec{S} t_{g}^{j}\right) \leq f\left(\vec{S}_{g}^{j}\right)\right) \\ \vec{S}_{g}^{j} & \text { otherwise }\end{cases}
$$

Where $\vec{S}_{g+1}^{j}, \vec{S}_{g}^{j}$ and $\vec{S}_{g}^{j}$ are the individual will be inserted into the new generation, the trial vector and the current population individual. The $\vec{S}_{g}^{j}$ is called target vector and it is the same vector in (14) and (15). According (15), the trial vector competes against the target vector. The vector with the lowest objective value survives into the next generation. The individual (target vector) form current generation survives into the next generation, if mutation and recombination did not improve its quality.

The elements of all vectors in (13), (14) and (15) are virtual node loads.

\section{The proposed method and experimental verification of thesis}

\subsection{Preview of the proposed method}

Figure 2 shows the flow chart of the method. In order to consider the most general case of power flow calculation in an asymmetric network, the method described in [14] is used for power flow calculation. 

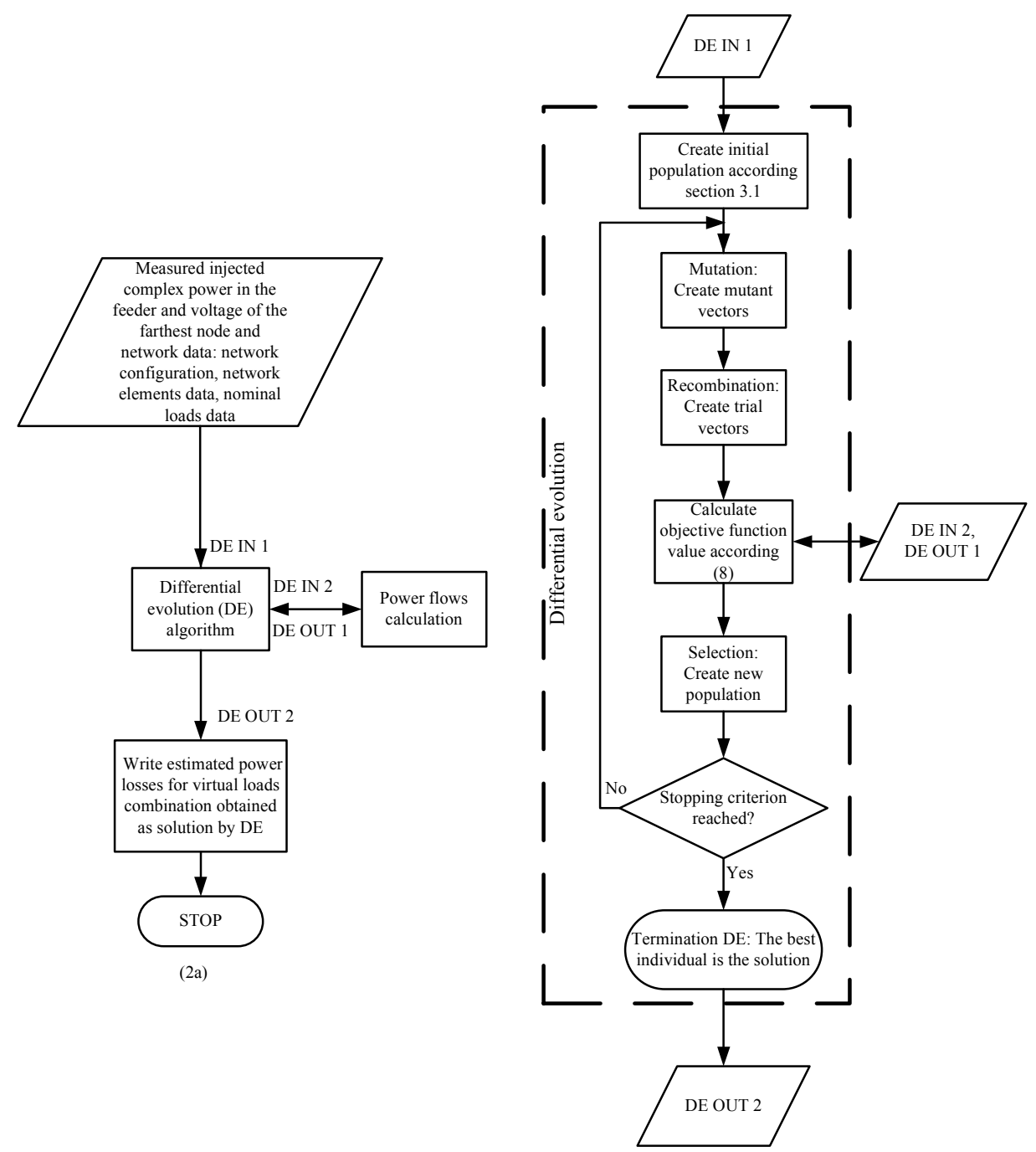

$(2 \mathrm{~b})$

Figure 2. Flow chart of the proposed method (2a) and DE algorithm (2b)

It needs to be emphasized at this point that during the performance of $\mathrm{DE}$, the power flow is calculated for every individual in the population. Power flow calculation determines the injected power, as well as the load of the farthest node required for calculation of the fitness function.

\subsection{Description of the calculation procedure}

In order to confirm the validity of the thesis presented in Part IV of this paper, further work is done with the DE algorithm described in Part V.

The process of performing calculations required for verification of the thesis is performed in the following steps:

Step 1 For a given combination of node loads, calculations of power flow are performed. 
International Journal of

Soft Computing And Software Engineering (JSCSE)

e-ISSN: 2251-7545

Vol.2,No.4, 2012

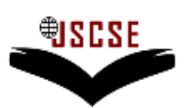

DOI: 10.7321/jscse.v2.n4.1

Step 2 Based on the calculation from Step 1, the total complex power injected into the feeder, total active losses and voltages on the feeder are determined.

Step 3 For the known injected power at the beginning of the feeder and known voltages of the farthest node from Step 2, virtual load combinations, which give approximately the same injected complex power at the beginning of the feeder and voltages in the farthest node are determined by DE.

Step 4 A comparison of the total power injected in the feeder, the total power of loads and the total losses on the feeder is performed for the given loads from Step 1 and virtual loads obtained in Step 3.

When performing the DE algorithm, the following parameters are used: the number of vectors in the population $N i=50$, number of generations $g=30$, constant of mutation $F=0.5$ and coefficient of recombination $\mathrm{Cr}=0.8$. According to [13], recommended values of parameters $\mathrm{F}$ and $\mathrm{Cr}$ are in interval of $F \in[0.5,1.0]$ and $C r \in[0.8,1.0]$.

The algorithms of the proposed method and DE shown in Fig.2, as well as power flow calculation according to [14] have been programmed in MATLAB 7.9.0.

\subsection{Comment on the Calculation Results}

The IEEE 123 node test feeder has been used as an example of a radial distribution feeder. Data for these networks are described in [15] and are available online [16]. The test results of the proposed method conducted on the IEEE 123 bus feeder are shown at the end of the paper (6. Appendix ).

For the purpose of carrying out the procedure presented in part 5.2., first several different node load combinations (Table 2, 6. Appendix) labeled as LC1-LC7 have been generated. The columns in Table 2 labeled as LC1-LC7 contain loads in which a specific percentage of the rated load is stated for each row of the table. These load combinations are generated by choosing the specific percentage of rated load for LC1, LC2, LC5-LC7. For LC3 and LC4 load combinations, the percentage of rated load is randomly determined within the specified range of the rated load. Loads generated in this way have been taken as real loads at some moment in the network. Power flow calculations have been performed for these loads. Moreover, total losses taken as real losses in the network have been calculated. Despite the total losses, real injected power and real voltage of the farthest node have been calculated, and have been used as input data.

The results obtained by the proposed method, as well as the results of scaled loads of a single phase using the ratio of real injected power and rated power of a single phase are shown in Table 3 (6. Appendix). During loss determination, loads scaled by the same percentage of rated power exhibit lack of precision (from $0.62 \%$ for LC3 to $24 \%$ for LC4) for different load combinations. Errors of loss estimation can account for $20 \%$ ( $24 \%$ for LC4). The reason for this lies in the fact that only injected power is taken into account. Since different load combinations can give approximately the same injected power, it is obvious why such great errors occur. This is particularly visible in the cases of LC2, LC3 and LC4 which all have a similar amount of total injected power in the network. Scaling of all loads using the same percentage of rated power for all three cases yields approximately the same losses. This problem may occur in all methods which only take input power into consideration.

The highest average error obtained by the proposed method is less than $6.5 \%$, which makes it over 14 times (for LC2) more accurate than loss estimation carried out by load scaling. The estimated losses, as well as voltages of the farthest node are close to real values for all presented load combinations.

Simulations for the test feeder have been performed in MATLAB 7.9.0. software. Simulations have been carried out using HP 6830s s Intel Core 2 Duo CPU P8400 2.26 GHz Memory (RAM) 4 GB.

Simulation time for the tested IEEE 123 network was 174 s. The main reason for such a long simulation time is the necessity to calculate the power flow for each individual, which, in our case, are 50 individuals $\times 30$ generations $=1500$ power flow calculations. Of course, in case of a smaller network the simulation time reduces. Furthermore, the calculation time can be reduced by using a faster computer and the possibility of parallel performance of the evolution algorithm in MATLAB software. 


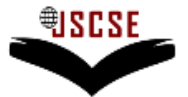

DOI: $10.7321 /$ jscse.v2.n4.1

\section{Conclusion}

This paper describes a procedure for estimation of total losses on a radial feeder of distribution networks using DE and power flow calculation. In this process, DE has been used by means of which certain combinations of virtual loads were found for the presented objective function. The objective function is defined on the basis of known injected complex power into the feeder and voltages in the farthest network node.

The proposed method has certain advantages over existing methods, i.e. only measurements of total complex power injected into the radial feeder and voltages in the farthest node are needed. Moreover, knowledge of load curves or statistical data considering load is not required. This reduces the impact of errors in the evaluation of losses due to load uncertainty. In addition, it is not necessary to model the load on more levels because the virtual amount of load in each node is determined using DE for the moment of measuring the injected power. Also, it is not necessary to perform the measurement and analysis of the dependence of power losses. Furthermore, the advantage of the proposed method is that the total complex power injected into the radial feeder and voltages of the farthest network node at a given time is in use. This allows the application of methods in almost real time compared to the moment of measuring the injected power. Time discrepancy occurs because of the time needed to calculate power flows in DE iterations.

Shortcoming of the proposed method stems from the need to know the parameters of all network elements and network configurations at the time of observation. In practice, problems may occur if it does not have data on the network or these data are unreliable. Also, preparation and input of all network elements require a lot of work. Since the DE is an iterative procedure and the power flow calculation is performed iteratively, problems in the duration of calculation on the computer can occur. These shortcomings may be particularly displayed in the case of large distribution networks. Therefore, the distribution network size (number of nodes, lines, loads) can be a limitation for the application of the proposed method. In addition to the long calculation time, problems may arise with quality of solutions. The number of loads is greater in larger networks. Since the loads are variables of the objective function, an individual (vector) in DE has more elements. Larger number (over a hundred in large distribution networks) of variables requires greater population and number of generations in DE and decreases quality of the solution.

The size and the configuration complexity of distribution networks for which the proposed method is applicable will be the subject of future work. Also, future research will be directed to determining the minimum required number of measurement data in larger networks. Tests with hybrid methods (heuristic-evolutionary, fuzzy-evolutionary, and others) will be included in future work. The application of hybrid method should be reduced the calculation time and improve solution quality.

Apart from the stated, the proposed method has shown that the use of the objective function for calculating losses (12) cannot result in only one load combination with approximately equal injected power and various losses. Therefore, methods which provide universal solutions for losses solely based on the power at the beginning of feeder cannot always result in reliable loss estimation. This is why a new variable was introduced, i.e. the amount of voltage at the farthest network node.

\section{Appendix}

The tested network is IEEE 123 node test feeder [16]. Tested load combinations are shown in Table 2. Results for the losses obtained for the proposed method and scaled loads are shown in Table 3 . The farthest three-phase node is node 95 . 
Table 2. Tested load combinations for IEEE 123 node test feeder

\begin{tabular}{|c|c|c|c|c|c|c|c|c|c|}
\hline \multirow{2}{*}{\multicolumn{3}{|c|}{$\begin{array}{c}\text { IEEE } 123 \text { node } \\
\text { feeder }\end{array}$}} & \multicolumn{7}{|c|}{ Load combination } \\
\hline & & & LC 1 & LC 2 & LC 3 & LC 4 & LC 5 & LC 6 & LC 7 \\
\hline \multirow{10}{*}{ 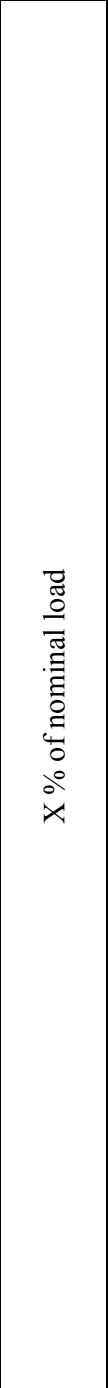 } & $10 \%$ & & $33,38,39,51$ & $\begin{array}{c}1-47,49,51, \\
53,56,59,74, \\
79,82,85,87, \\
90,94,96,99, \\
102,104,107, \\
111,113\end{array}$ & - & - & - & - & - \\
\hline & $20 \%$ & & \begin{tabular}{|c|}
$1,5,6,9,11,12$, \\
$17,24,29-31,34$, \\
$35,41,45,47,48$, \\
$50,52,55,58,60-$ \\
$63,66,70,74,77$, \\
$79,82,86,100$, \\
$102-104$
\end{tabular} & - & - & - & $\begin{array}{c}5,79,82,107 \\
111,114\end{array}$ & - & - \\
\hline & $30 \%$ & & \begin{tabular}{|c|}
$2,7,10,16,19$, \\
$22,28,32,37,42$, \\
$46,49,53,56,64$, \\
$68,69,71,80,83$, \\
$85,87,90,99$
\end{tabular} & - & - & - & $\begin{array}{c}10,55,70,73 \\
75,83,86,88 \\
94,98,104,109 \\
112\end{array}$ & $\begin{array}{c}1,2,6-9,24,28 \\
38-41,48,50 \\
64,100,102\end{array}$ & - \\
\hline & $40 \%$ & & $\begin{array}{c}4,43,59,73,75 \\
76,84,88\end{array}$ & - & - & - & \begin{tabular}{|c|}
$62,64,74,84$ \\
$90,95,100,103$ \\
113 \\
\end{tabular} & $\begin{array}{c}5,11,17,34,58, \\
66\end{array}$ & - \\
\hline & $50 \%$ & $\frac{0}{0}$ & 65,80 & - & - & - & $\begin{array}{c}20,42,63,65 \\
66,96,102,106\end{array} \mid$ & $\begin{array}{c}4,12,19-22,29, \\
33,43,47,49, \\
111,112\end{array}$ & - \\
\hline & $60 \%$ & 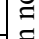 & - & - & - & - & $33,50,60,68$ & 76 & - \\
\hline & $70 \%$ &.$\Xi$ & - & - & - & - & $11-17$ & - & $\begin{array}{c}1-28 \\
76\end{array}$ \\
\hline & $80 \%$ & & 98 & - & - & - & $29,41,53$ & $\begin{array}{c}73,86,96,106, \\
107\end{array}$ & $29-46$ \\
\hline & $90 \%$ & & - & - & - & - & $\begin{array}{c}19,28,35-39 \\
52,76\end{array}$ & $\begin{array}{c}16,30,60,62, \\
82,84,87,90, \\
95,103\end{array}$ & $\begin{array}{l}51-75 \\
77-95\end{array}$ \\
\hline & $100 \%$ & & $\begin{array}{c}92,94,95,96 \\
106,107,109 \\
111,114\end{array}$ & $\begin{array}{c}48,50,52,55 \\
58,60-73,75- \\
77,80,83,84, \\
86,88,92,95, \\
98,100,103 \\
106,109,112 \\
114\end{array}$ & - & - & $\begin{array}{c}1-4,6-9,22,24 \\
30-32,34,43-49 \\
56-59,69,71 \\
77,80,85,87 \\
92\end{array}$ & $\begin{array}{c}10,31,32,35, \\
37,42,45,46, \\
51-56,59,63,65\end{array}$ & $\begin{array}{c}47-50 \\
96- \\
114\end{array}$ \\
\hline \multirow{4}{*}{ 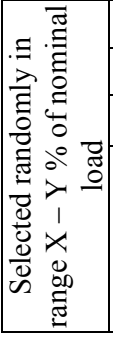 } & $10-20 \%$ & & - & - & - & $1-50$ & - & - & - \\
\hline & $10-30 \%$ & & - & - & $51-114$ & - & - & - & - \\
\hline & $20-50 \%$ & & - & - & - & $73-79$ & - & - & - \\
\hline & $\begin{array}{c}80-100 \\
\%\end{array}$ & & - & - & $1-50$ & $\left|\begin{array}{l}51-71 \\
80-114\end{array}\right|$ & - & - & - \\
\hline
\end{tabular}


Table 3. Results for IEEE 123 node test feeder

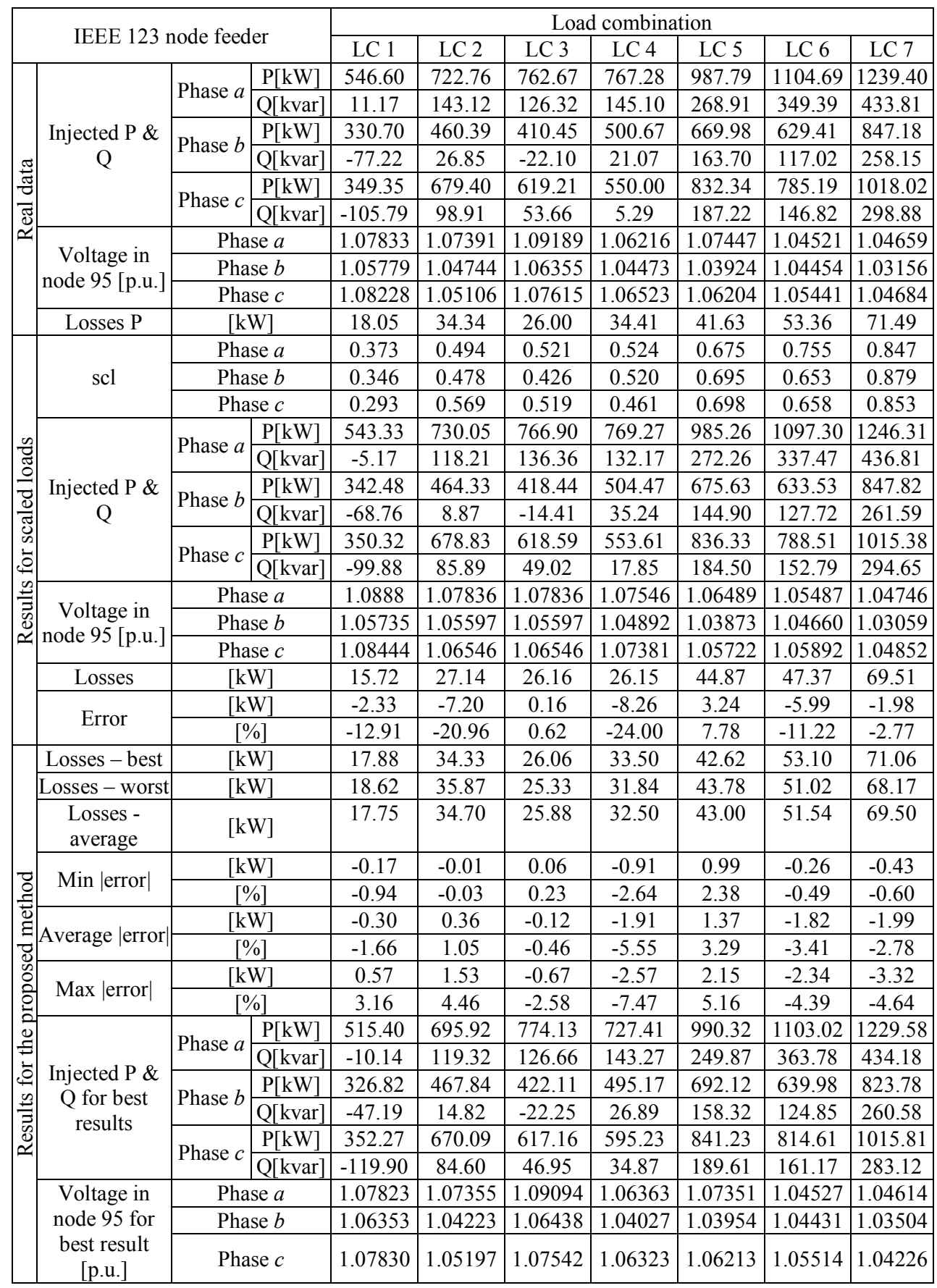




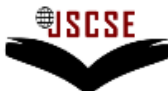

DOI: $10.7321 /$ jscse.v2.n4.1

\section{References}

[1] D. L. Flaten, "Distribution System Losses Calculated by Percent Loading", IEEE Transactions on Power Systems, vol. 3, no. 3, pp. 1263-1269, 1988.

[2] M. W. Gustafson, J. S. Baylor and S. S. Mulnix, "The Equivalent Hours Loss Factor Revisited", IEEE Transactions on Power Systems, vol. 3, no. 4, pp. 1502-1508, 1988.

[3] M. W. Gustafson and J. S. Baylor, "Approximating the System Losses Equation”, IEEE Transactions on Power Systems, vol. 4, no. 3, pp. 850-855, 1989.

[4] R. Baldick and F. F. Wu, "Approximation Formulas for the Distribution System: the Loss Function and Voltage Dependence", IEEE Transactions on Power Delivery, vol. 6, no. 1, pp. 252-259, 1991.

[5] C. S. Chen, M. Y. Cho and Y. W. Chen, "Development of simplified loss models for distribution system analysis", IEEE Transactions on Power Delivery, vol. 9, no. 3, pp. 1545-1551, 1994.

[6] H-D. Chiang, J-C. Wang and K. N. Miu, "Explicit Loss Formula, Voltage Formula and Current Flow Formula for Large-Scale Unbalanced Distribution Systems", IEEE Transactions on Power Systems, vol. 12, no. 3, pp. 1061-1067, 1997.

[7] Roytelman and S. M. Shaihidehpour, "State estimation for electric power distribution systems in quasi real-time conditions", IEEE Transactions on Power Delivery, vol. 8, no. 4, pp. 2009-2015, 1993.

[8] Y-Y. Hong and Z-T. Chao, "Development of Energy Loss Formula for Distribution Systems Using FCN Algorithm and Cluster-Wise Fuzzy Regression”, IEEE Transactions on Power Delivery, vol. 17, no. 3, pp. 794-799, 2002.

[9] Y-Y. Hong, Z-T. Chao and M-S. Yang, "A fuzzy multiple linera regression based loss formula in electric distribution systems", Fuzzy Sets and Systems, vol.142, no.2, pp. 293-306, 2004.

[10] V. Mogilenko and D. A. Pavlyuchenko, "The Estimation of Electric Power Losses in Electrical Networks by Fuzzy Regression Model Using Genetic Algorithm”, Mathware \& Soft Computing, vol. 11, pp. 13-30, 2004.

[11]Dortolina and R. Nadira, "The Loss That is Unknown is No Loss At All: A Top-Down/Bottom-Up Approach for Estimating Distribution Losses”, IEEE Transactions on Power Systems, vol. 20, no. 2, pp. 1119-1125, 2005.

[12]P. S. N. Rao and R. Deekshit, "Energy Loss Estimation in Distribution Feeders", IEEE Transactions on Power Delivery, vol. 21, no. 3, pp. 1092-1100, 2006.

[13]U. K. Chakraborty (Ed.), Advances in Differential Evolution, Heidelberg: Springer, 2008.

[14] T-H. Chen and N-C. Yang. (2010, February 2), "Loop frame of references based three-phase power flow for unbalanced radial distribution systems", Electric Power Systems Research, vol. 80, no. 7, pp. 799-806, 2010.

[15] W. M. Kersting, "Radial Distribution Test Feeders", in Proceedings 2001 IEEE Power Engineering Society Winter Meeting, vol. 2, pp. 908-912, 2001.

[16] IEEE PES (2010, June 10), [Online]. Available: http://ewh.ieee.org/soc/pes/dsacom/testfeeders/index.ht 